\title{
Cyclone-Anticyclone Asymmetry in Geophysical Turbulence
}

\author{
Guillaume Roullet* and Patrice Klein ${ }^{\dagger}$ \\ Laboratoire de Physique des Océans UMR6523 (CNRS, UBO, IFREMER, IRD), IFREMER, Brest, France
}

(Received 14 December 2009; published 25 May 2010)

\begin{abstract}
We address the problem of cyclone-anticyclone asymmetry in geophysical turbulence using a direct numerical simulation with high Reynolds number $\mathrm{Re} \sim 15000$ that includes an active upper boundary and interior dynamics. The regime, characterized by a finite Rossby number (Ro $\sim 0.6)$ strongly departs from the classical quasigeostrophic regime. The numerical resolution is pushed to the limit of today's supercomputer capabilities ensuring more than two decades free of viscous effects. The results show a strong cyclonic dominance in the upper layers that is stronger for filaments than for vortices. This is in contrast with similar studies that have no active upper boundary which reported either anticyclone dominance or a symmetry between cyclones and anticyclones in the upper layers. This highlights the impact of boundary dynamics on geophysical turbulence.
\end{abstract}

DOI: 10.1103/PhysRevLett.104.218501

PACS numbers: 92.10.ak, 47.27.E-

Coherent vortices are known to emerge in geophysical turbulent flows under the influence of planetary rotation and stable density stratification. The regime is characterized by the Rossby and Burger numbers [respectively defined as $\mathrm{Ro}=U / f L$ and $\mathrm{Bu}=\left(L_{d} / L\right)^{2}$, with $U$ and $L$ the horizontal velocity and length scales, $f$ the Coriolis frequency, $L_{d}=N H / f$ the deformation radius, $H(H \ll$ $L)$ the vertical scale, and $N(N \gg f)$ the stratification strength] $[1,2]$. Because of the nonlinear interactions [24], the spatial scales of these vortices tend to spontaneously adjust towards a regime for which $\mathrm{Bu}=\mathcal{O}(1)$. The resulting turbulence, usually sustained by the baroclinic instability of a large-scale vertically sheared flow (intensified near the upper boundary) and damped by bottom friction, is governed by the conservation of potential vorticity (PV) $[1,2]$. In the quasigeostrophic limit $($ Ro $\ll 1)$ a strict symmetry between cyclones and anticyclones is found $[1,2]$. This symmetry is usually broken by finite Ro number effects as revealed by numerical simulations [5-8] with a dominance of anticyclones. These simulations, however, involve no active boundary since they ignore the impact of boundary density anomalies (and the associated frontogenesis). As such, this turbulence can be described in terms of interior PV dynamics. An example of a study that includes the effects of boundary dynamics (but ignores interior PV) reports in contrast a cyclonic dominance [9]. It is not yet clear how boundary density anomalies interact with interior PV anomalies [10]. The impact is often assumed to be negligible [11]. To further understand this interaction we use a direct numerical simulation of forced geophysical turbulence that involves both boundary density and interior PV anomalies and show how the cycloneanticyclone asymmetry varies in the vertical.

The direct numerical simulation is computed by integration of the following primitive equations [12], derived from the Navier-Stokes equations using the Boussinesq and hydrostatic (since $H \ll L$ ) approximations that are appli- cable for geophysical flows,

$$
\begin{gathered}
D_{t} u=-\partial_{x} \phi+(f+\beta y) v+F_{u}+D_{u}, \\
D_{t} v=-\partial_{y} \phi-(f+\beta y) u+F_{v}+D_{v}, \\
0=-\partial_{z} \phi+b, \\
0=\partial_{x} u+\partial_{y} v+\partial_{z} w \\
D_{t} b=F_{b}+D_{b},
\end{gathered}
$$

with $D_{t}=\partial_{t}+u \partial_{x}+v \partial_{y}+w \partial_{z}$ the material derivative, $(u, v, w)$ the three components of the velocity, $\phi$ the pressure, $b$ the buoyancy (rescaled density), and $\beta$ the local variation of $f . F_{(u, v, b)}$ and $D_{(u, v, b)}$ are the forcing term and the damping or dissipation term, respectively (see below). The numerical methods used to integrate these equations are described in [13]. The geometry is an $x$-periodic channel (1000 km long, $3000 \mathrm{~km}$ wide, $4000 \mathrm{~m}$ deep) centered at midlatitudes $\left(f=10^{-4} \mathrm{~s}^{-1}, \quad \beta=1.6 \times\right.$ $10^{-11} \mathrm{~s}^{-1} \mathrm{~m}^{-1}$ ). The model resolution is $\Delta x \sim 1 \mathrm{~km}$ in the horizontal and with 200 stretched vertical levels (see Fig. 1). The vertical stratification is intensified in the upper $1000 \mathrm{~m}$ and corresponds to a deformation radius $L_{d}=$ $30 \mathrm{~km}$, typical of an oceanic midlatitude regime (Fig. 1). Vortices emerge from the baroclinic instability of a largescale sheared flow $\left[u^{*}(y, z), v^{*}=0\right]$ in thermal wind balance with a large-scale meridional buoyancy $b^{*}(y, z)=$ $\bar{b}(z)+F(y) \tilde{b}(z)$ (Fig. 1) that extends from the upper boundary down to $1500 \mathrm{~m}$ in the interior. The turbulence is forced by restoring to the basic state jet $F_{u}=\left(u^{*}-\right.$ $\left.\bar{u}^{x}\right) / \tau$ (and accordingly for $v$ and $b$ ), where $\bar{u}^{x}$ denotes the $x$ average and $\tau=50$ days. The damping is performed by a bottom Ekman friction $-\lambda(u, v) \delta(z) \quad[\lambda=4.6 \times$ $10^{-4} \mathrm{~m} \mathrm{~s}^{-1}$ and $\delta(z)$ is the Dirac-delta function at the bottom]. Dissipation involves a bi-Laplacian on the hori- 


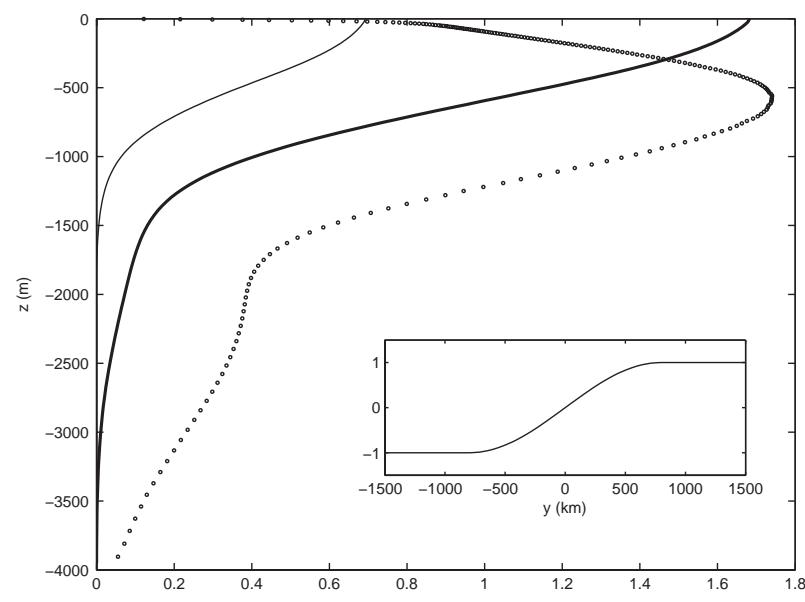

FIG. 1. Vertical profiles of the horizontal mean stratification $\bar{b}(z) \quad$ (thick line), its associated buoyancy frequency $N(z) H /\left(\pi f L_{d}\right)$ (one circle per $z$ level), and the jet buoyancy anomaly $\tilde{b}(z)$ (thin line) for the surface intensified experiment. Buoyancy is normalized by $H /\left(\pi f L_{d}\right)^{2}$. The meridional structure function $F(y)$ of the jet is plotted in the inset; its buoyancy profile is $b^{*}(y, z)=\bar{b}(z)+F(y) \tilde{b}(z)$.

zontal $K \nabla^{4}\left(K=2 \times 10^{6} \mathrm{~m}^{4} \mathrm{~s}^{-2}\right)$ and a constant vertical diffusion coefficient $\left(10^{-5} \mathrm{~m} \mathrm{~s}^{-1}\right)$. The simulation is integrated up to statistical equilibrium (600 days). The flow displays a large Reynolds number $(\operatorname{Re} \sim 15000$, computed using an equivalent viscosity $\nu=K / \Delta x^{2}, U=0.15 \mathrm{~m} / \mathrm{s}$, and $L=200 \mathrm{~km}$ ) and a finite Rossby number in the upper layers $(\mathrm{Ro}=0.6)$. Spectral characteristics of this simulation reveal a boundary regime distinct from the deep interior regime with a smooth transition in between [14]. At the boundary, the $k^{-2}$ velocity spectrum well matches the density spectrum when the latter is appropriately scaled [14] (blue and red curves, respectively, in Fig. 2) and emphasizes the emergence of small scales (submesoscales). The velocity spectrum in the interior has a much steeper slope $\left(k^{-3.5}\right)$ (green curve in Fig. 2) and the density spectrum (not shown) an even steeper slope $\left(k^{-4.5}\right)$. These spectral properties in the interior are close to those reported for quasigeostrophic regimes $[4,15]$. The relative vorticity (defined as $\zeta=v_{x}-u_{y}$ ) involves large values, from $-f$ to $3 f$ at the upper boundary (Fig. 3). The flow consists of numerous small-scale vortices as well as thin filaments both with quite large vorticity values, whereas at $800 \mathrm{~m}$ the relative vorticity field is dominated by larger (mesoscale) vortices (not shown).

In this Letter, we focus on the vortical asymmetry. The asymmetry is examined using higher order moments of $\zeta / f$ as a function of depth (Fig. 4). The skewness of $\zeta / f$ (black curve in Fig. 4) reveals near the upper boundary a significant vortical asymmetry and, in particular, a dominance of cyclonic structures in the first $600 \mathrm{~m}$. The skewness is almost constant (larger than 1.3) from the upper boundary down to $300 \mathrm{~m}$ before decreasing to zero at $600 \mathrm{~m}$. It becomes negative between 600 and $1100 \mathrm{~m}$ in

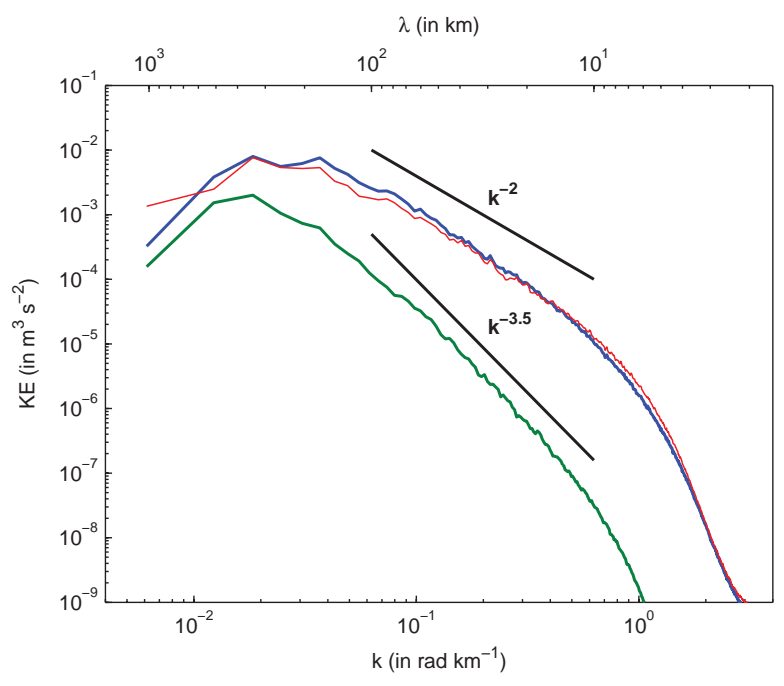

FIG. 2 (color). Velocity spectrum (blue line) and density spectrum (scaled to match the velocity spectrum, see [14]) (red line) at the upper boundary. Velocity spectrum at $800 \mathrm{~m}$ (green line). Horizontal axis displays the wave number $k$ and wavelength $\lambda$.

a region where kinetic energy (KE) values are smaller. The large positive skewness values near the boundary are accompanied by a local increase of the kurtosis (red curve in Fig. 4). The region of cyclonic dominance $(600 \mathrm{~m}) \mathrm{con}-$ tains more than $50 \%$ of the total kinetic energy. The probability density function (PDF) of $\zeta / f$ (Fig. 4) near the boundary is close to an exponential tail for cyclonic structures and a Gaussian one for anticyclonic structures, with a monotonic decrease with depth.

Relative vorticity structures involve both coherent vortices (with circular patterns) and filaments exhibiting

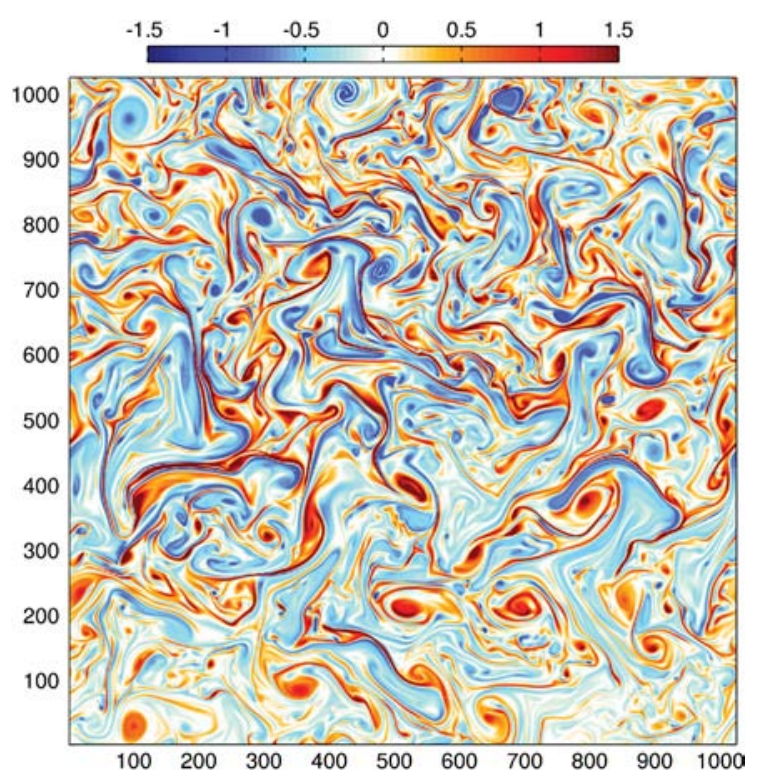

FIG. 3 (color). Snapshots of $\zeta / f$ at the upper boundary. Color scale range is chosen to better emphasize the vorticity patterns. 


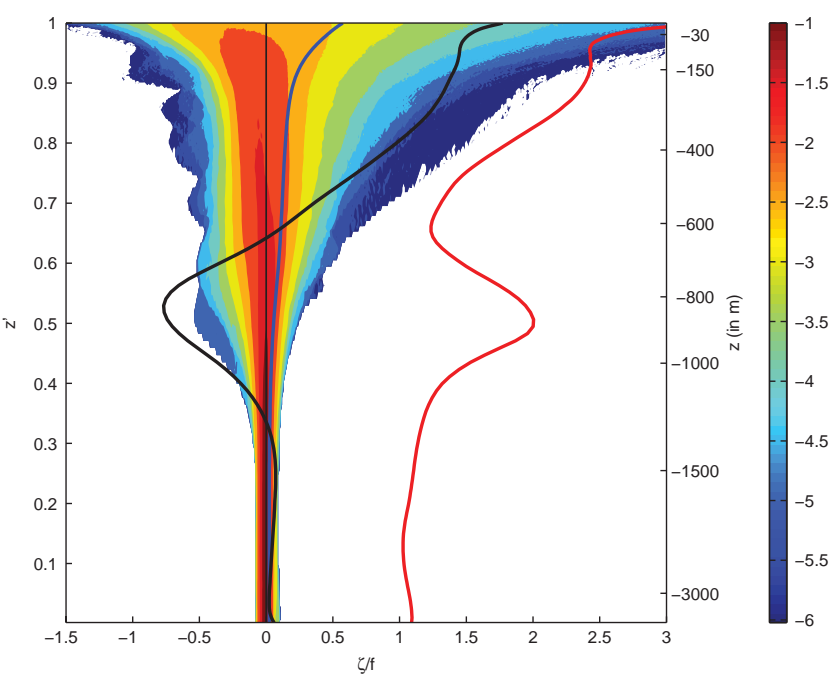

FIG. 4 (color). Isocontours of $\log _{10} p(\zeta / f, z)$ with $p(\zeta / f, z)$ the PDF of $\zeta / f$ as a function of depth. The three superimposed curves are, respectively, the rms (blue), the skewness (black), and the kurtosis divided by three (red) of $\zeta / f$. The vertical axis is, on the left-hand side, the nondimensional stretched vertical coordinates [such that $d z^{\prime}=N(z) d z / f L_{d}$, see [3]], and on the right-hand side, the corresponding dimensional depth.

small-scale elongated structures. Different geometrical methods exist to partition vortices from filaments, such as vortex census algorithms [9] or the wavelet analysis methods [16]. Most of them are valid for freely decaying turbulence and do not work so well for forced turbulence where vortices are permanently deformed. In the present study we have chosen to use the classical Okubo-Weiss (OW) criterion [17] [defined as $\left(\sigma^{2}-\zeta^{2}\right) / f^{2}$, with $\sigma=$ $\sqrt{\left(u_{x}-v_{y}\right)^{2}+\left(v_{x}+u_{y}\right)^{2}}$ the strain field] that quantifies the magnitude of $\zeta$ relative to $\sigma$. The magnitude of $\zeta$ within vortices is usually much larger than $\sigma$, which allows them to remain coherent and persistent in time [18]. On the other hand, the magnitude of $\zeta$ within the filaments is usually close to or smaller than $\sigma$ because of their elongated pattern [18], which causes them to deform rapidly. We have chosen to define vortices as the structures where the OW criterion is smaller than a given value $(-0.05$ at the upper boundary and -0.005 at $800 \mathrm{~m}$ ) and define filaments as the structures for which OW is larger than these values. These values roughly correspond to the vortices occupying $20 \%$ of the total area. The selected structures have principally a circular pattern and almost all of the eliminated structures (not shown) are stretched filaments. The sensitivity of the results discussed below to the choice of these values has been found to be very weak.

Figure 5 show the PDFs of $\zeta / f$ for vortices and filaments. At the upper boundary [Fig. 5(a)] both vortices and filaments display a strongly asymmetric PDF indicating cyclonic dominance. The skewness of the filaments (1.96) is conspicuously larger than that for vortices (1.16). This
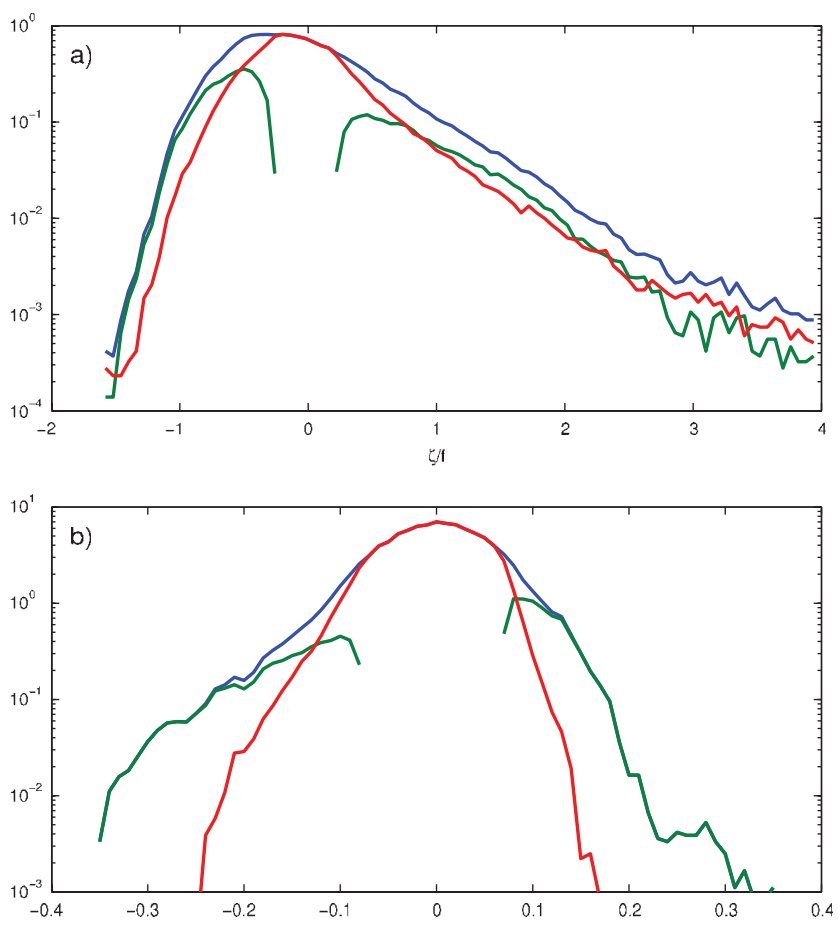

FIG. 5 (color). PDF of $\zeta / f$ at the upper boundary (top) and $800 \mathrm{~m}$ (bottom). The blue curve is the total PDF. The green (red) curve is the PDF of the relative vorticity related to the vortices (filaments); see text for the partitioning.

difference decreases with depth, and at $400 \mathrm{~m}$ the skewness is 0.5 for filaments and 0.36 for vortices. At $800 \mathrm{~m}$ the PDFs are almost symmetric with the PDF of filaments almost Gaussian [red curves in Fig. 5(b)]. Skewness for filaments and vortices is smaller and close $(\approx-0.3)$. These results clearly confirm the strong differences between boundary and interior regimes and highlight the stronger vortical asymmetry for filaments than for vortices near the upper boundary.

Our numerical primitive equations solution shows strong cyclonic dominance for both vortices and filaments. This is different from similar studies that have no active boundary which reported either anticyclonic dominance or a symmetry between cyclones and anticyclones [5-8,19]. To further highlight the impact of an active surface boundary, we performed an additional simulation similar to the previous one but with the large-scale meridional density gradient set to zero very close to the surface boundary (such that there is no surface density anomaly). Although the KE is still intensified in the first $500 \mathrm{~m}$ with almost the same magnitude, no boundary regime emerges in this new simulation: the velocity spectrum is characterized by a steep $k^{-3.5}$ slope in the upper layers indicating much weaker submesoscales. In these layers the skewness of $\zeta / f$ is close to zero, clearly indicating a symmetry between cyclones and anticyclones, and the PDF of $\zeta / f$ is almost symmetric with the magnitude of $\zeta / f$ smaller than 0.5 . These very different results suggest that the source of vortical asym- 
metry is related to the specific boundary regime that includes energetic submesoscales and associated frontogenesis.

These results question the mechanisms through which boundary density anomalies produce a strong cyclonic dominance and what explains the depth extension of this asymmetry. To delve deeper into the first question we can focus on the stronger vortical asymmetry (with cyclonic dominance) for filaments than for vortices observed in our solution. Reports of such strong filament vortical asymmetry are not new [9]. It has been explained [20,21] as the ageostrophic frontogenesis impact on the boundary density filaments. We speculate that this strong filament vortical asymmetry may be one source of the cyclonic vortex dominance through the following mechanisms: the larger vorticity amplitude in cyclonic filaments make them to be more unstable (in terms of shear instability [22]) than anticyclonic filaments, leading to a larger production of small-scale cyclonic vortices. By invoking the inverse KE cascade that covers a large spectral range at the boundary $[14,23]$, this should lead to a stronger flux of cyclones, leading to cyclonic vortex dominance at larger scales. This conjecture may indicate an intensification of the transformation of available potential energy into KE-and therefore of the total KE-because of this specific boundary dynamical impact. However, this conjecture has still to be verified, and will be addressed in a future work.

Our solution further reveals that the boundary dynamics extends well into the interior. This depth extension of the cyclone dominance seems to indicate a coupling between boundary and interior dynamics [24] more complex than suggested $[11,3]$ and that may involve mixed boundaryinterior instabilities [25]. Attempts to understand this coupling $[24,25]$ should be taken further to better understand its intensity in relation with the large-scale properties of the flow such as the background stratification.

The authors thank S. Smith, A Bracco, and the reviewers for their valuable comments. This work is supported by IFREMER, CNRS (France), the Agence Nationale pour la Recherche (Contract No. ANR-05-CIGC-010). Numerical simulations reported here were done on the Earth Simulator (Yokohama, Japan) through a M.O.U. between IFREMER, CNRS, and JAMSTEC. *roullet@univ-brest.fr

†patrice.klein@ifremer.fr

[1] J. Pedlosky, Geophysical Fluid Dynamics (SpringerVerlag, Berlin, 1987).

[2] J. C. McWilliams, J. B. Weiss, and I. Yavneh, Science 264, 410 (1994).

[3] J. Charney, J. Atmos. Sci. 28, 1087 (1971).

[4] B. L. Hua and D. B. Haidvogel, J. Atmos. Sci. 43, 2923 (1986).

[5] L. M. Polvani, J. C. McWilliams, M. A. Spall, and R. Ford, Chaos 4, 177 (1994).

[6] J. Y.-K. Cho and L. M. Polvani, Phys. Fluids 8, 1531 (1996).

[7] I. Yavneh, A. F. Shchepetkin, J. C. McWilliams, and L. P. Graves, J. Comput. Phys. 136, 245 (1997).

[8] I. Koszalka, A. Bracco, J.C. McWilliams, and A. Provenzale, J. Geophys. Res. 114, C08011 (2009).

[9] G. J. Hakim, C. Snyder, and D. J. Muraki, J. Atmos. Sci. 59, 2405 (2002).

[10] S. K. Smith and R. Tulloch, J. Atmos. Sci. 66, 1073 (2009).

[11] E. Lindborg, J. Atmos. Sci. 66, 1069 (2009).

[12] J.R. Holton, An Introduction to Dynamic Meteorology (Academic, New York, 1992).

[13] A. F. Shchepetkin and J. C. McWilliams, Ocean Modelling 9, 347 (2005).

[14] P. Klein, B. Hua, G. Lapeyre, X. Capet, S. L. Gentil, and H. Sasaki, J. Phys. Oceanogr. 38, 1748 (2008).

[15] W. Blumen, J. Atmos. Sci. 35, 774 (1978).

[16] G. Lapeyre and P. Klein, J. Mar. Res. 64, 835 (2006).

[17] J. Weiss, Physica (Amsterdam) 48D, 273 (1991).

[18] B. L. Hua and P. Klein Physica (Amsterdam) 113D, 98 (1998).

[19] M. Arai and T. Yamagata, Chaos 4, 163 (1994).

[20] G. Lapeyre, P. Klein, and B. L. Hua, J. Phys. Oceanogr. 36, 1577 (2006).

[21] J. McWilliams, F. Colas, and M. J. Molemaker, Geophys. Res. Lett. 36, L18602 (2009).

[22] I. M. Held, R. T. Pierrehumbert, S. T. Garner, and K. L. Swanson, J. Fluid Mech. 282, 1 (1995).

[23] X. Capet, J.C. McWilliams, M. Molemaker, and A. Shchepetkin, J. Phys. Oceanogr. 38, 2256 (2008).

[24] G. Lapeyre and P. Klein, J. Phys. Oceanogr. 36, 165 (2006).

[25] R. Tulloch and S. K. Smith, J. Atmos. Sci. 66, 450 (2009). 